03

\title{
Механическая модель колебательного спектра углекислого газа
}

\author{
() Г.Т. Алдошин, С.П. Яковлев
}

Балтийский государственный технический университет „ВОЕНМЕХ“ им. Д.Ф. Устинова, 190005 Санкт-Петербург, Россия

e-mail: yakovlev_sp@mail.ru

(Поступило в Редакцию 23 ноября 2015 г. В окончательной редакции 10 мая 2016 г.)

Методами классической динамики исследованы нелинейные колебания молекулы $\mathrm{CO}_{2}$. В дополнение к ранее рассмотренному ангармонизму валентного угла, позволившему объяснить резонанс Ферми, учтена физическая нелинейность силового поля („жесткость“ и „мягкость“ пружин). В приближении „дальних соседей“ (учтены взаимодействия атомов кислорода) выведены уравнения нелинейной системы дифференциальных уравнений в форме Лагранжа. Методом инвариантной нормализации получено их аналитическое решение. Численным моделированием установлено возникновение в системе странного аттрактора. Даны рекомендации по выбору начальных условий, устраняющие возможность перехода регулярных биений в хаотические.

\section{Введение}

В газообразном состоянии вещества отсутствует упорядоченность молекул и, исключая моменты столкновений, молекулы движутся независимо. Поступательные и вращательные движения молекул, а также колебательные движения атомов внутри молекул можно считать независимыми от действия других молекул. Поэтому при исследовании газообразного состояния наибольший интерес представляет поведение индивидуальных молекул.

Ньютонова механика успешно применяется в кинетической теории газов. Но принято считать, что области применимости классической механики ограничены размерами, которые не слишком малы. Однако и в диапазоне размеров порядка межатомных расстояний классические представления не полностью теряют свое значение. Понятие „материальной точки“ как объекта, обладающего инертной массой, лишенного внутренней структуры, размеры которого много меньше характер- ного размера рассматриваемой задачи, не ограничено конкретными оценками. Поэтому тяжелые по сравнению с электронами атомные ядра во многих задачах можно рассматривать как классические материальные точки. Молекула рассматривается как динамическая система, имеющая постоянный состав, т.е. одинаковое число атомов, объединенных связями, которые моделируются невесомыми пружинами и которые могут совершать колебательные движения относительно равновесной конфигурации. Третий закон Ньютона накладывает ограничения на силы, с которыми две частицы действуют друг на друга: силы должны быть направлены по прямой, проходящей через частицы, в противоположные стороны и иметь одну и ту же величину. Закон не позволяет рассматривать электродинамические взаимодействия, кроме простого притяжения и отталкивания Кулона. Поэтому могут рассматриваться только „статические“ силы, зависящие от взаимного положения материальных точек. Таким образом, классическая механика, оставаясь в пределах механики Ньютона, Лагранжа, Гамильтона,

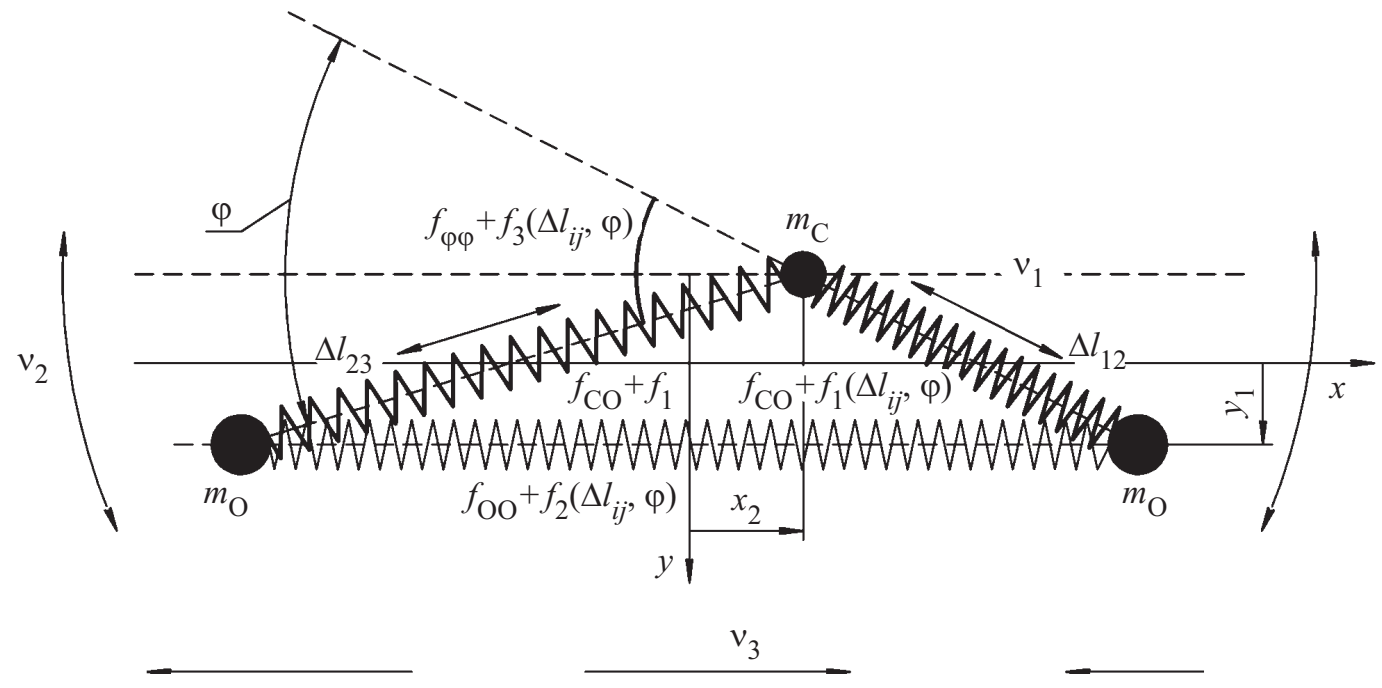

Рис. 1. Расчетная схема. 
позволяет изучать и объяснять механические эффекты колебаний атомов в молекуле.

Теория малых линейных колебаний разработана достаточно детально $[1,2]$. В линейном приближении изучаются только самые активные линии спектра. Введение геометрической нелинейности (ангармонизма валентного угла) [3] существенно изменяет всю картину движения: возникает сильное взаимодействие между модами колебаний, сопровождающееся периодическим обменом („перекачкой“) энергией между ними и расщеплением основной частоты рамановского спектра. Это явление, открытое в 1931г. Разетти и трактовку которого с квантово механических позиций в том же году дал Ферми [4], известно как резонанс Ферми.

Естественно ожидать новых эффектов в колебаниях молекулы, если учесть в ее механической модели дополнительные источники ангармонизма: взаимодействие атомов кислорода между собой (приближение „дальних соседей“") и физическую нелинейность силового поля $\mathrm{CO}_{2}$ („жесткость“ либо „мягкость““ пружин) [5].

\section{1. Постановка задачи и основные уравнения}

Рассматриваются свободные колебания молекулы идеального газа. Расчетная модель колебаний и основные обозначения:

- $m_{\mathrm{O}}, m_{\mathrm{C}}$ - массы атомов $\mathrm{C}, \mathrm{O}$ как материальных точек;

- $\mathrm{O}\left(x_{1}, y_{1}, 0\right), \mathrm{C}\left(x_{2}, y_{2}, 0\right), \mathrm{O}\left(x_{3}, y_{3}, 0\right)$ - текущие положения атомов;

- $v_{1}$ - линеаризованная собственная частота, соответствующая симметричной относительно центра массмолекулы форме колебаний, $\vartheta_{1}, v_{2}$ - частота деформационных или изгибных колебаний молекулы $\vartheta_{2}, v_{3}-$ частота антисимметричных или кососимметричных, валентных колебаний $\vartheta_{3}$ аналогичны [3] (рис. 1).

В дополнение к [3] в рассматриваемой модели учтена нелинейность сил взаимодействия атома углерода с атомами кислорода, а также атомов кислорода между собой:

- $f_{\mathrm{CO}}$ - линейная часть жесткости невесомой пружины, моделирующей межатомную связь С-О (валентной жесткости $), f_{1}\left(\Delta l_{i j}, \varphi\right)$ - нелинейная составляющая жесткости $\left(\Delta l_{i j}-\right.$ изменения расстояний между $i$-м и $j$-м атомами, $i \neq j ; \varphi-$ изменение валентного угла ОСO);

- $f_{\mathrm{OO}}$ - линейная часть жесткости пружины, моделирующей взаимодействие „дальних соседей“, $f_{2}\left(\Delta l_{i j}, \varphi\right)$ - ее нелинейная составляющая;

- $\left(f_{\varphi \varphi}+f_{3}\left(\Delta l_{i j}, \varphi\right)\right)$ - деформационная (изгибная) жесткость; поправочные коэффициенты приняты согласно экспериментальным данным [5].

Безразмерный гамильтониан системы в нормальных координатах $\bar{\vartheta}_{1}=\left(\bar{x}_{3}-\bar{x}_{1}\right) / 2$ (симметричная), $\bar{\vartheta}_{2}=\bar{y}_{1}$

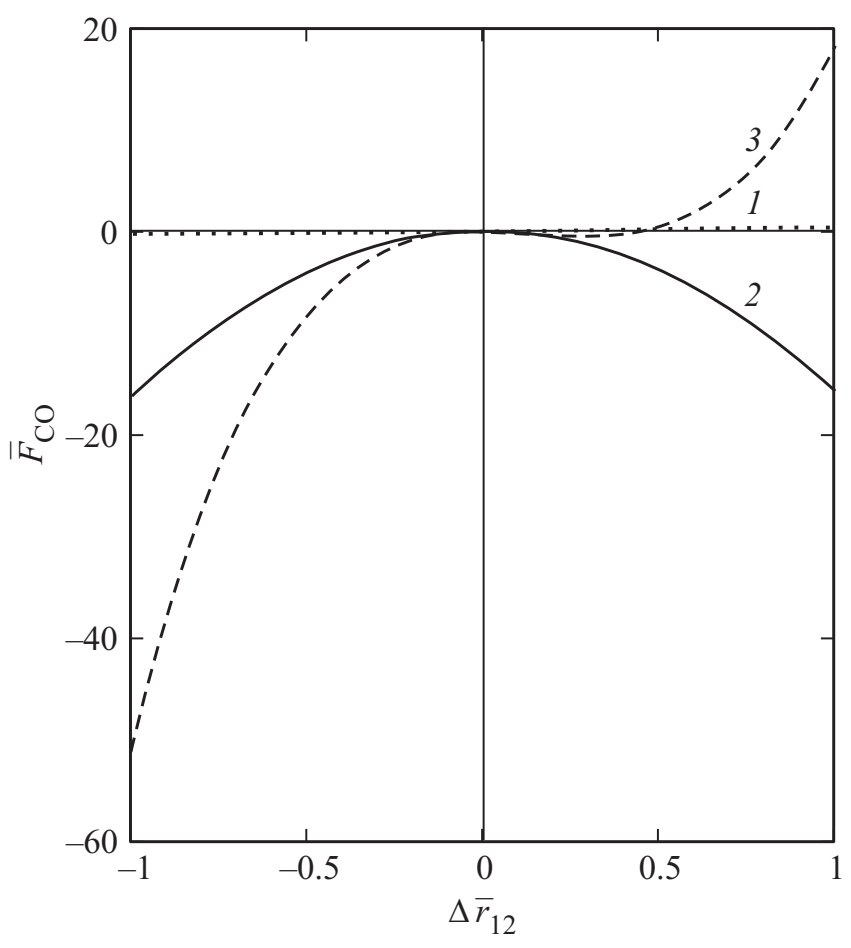

Рис. 2. Упругая характеристика межатомной связи С-O: 1 - линейное приближение (закон Гука), 2 - квадратичное приближение, 3 - кубичное приближение.

(деформационная), $\bar{\vartheta}_{3}=\left(\bar{x}_{1}+\bar{x}_{3}\right) / 2$ (антисимметричная) $\left(\bar{x}_{1}=\left(x_{1}-l\right) / l ; \bar{x}_{3}=\left(x_{3}+l\right) / l ; \bar{y}_{1}=y_{1} / l\right.$ : масштаб длины принят равным равновесной длине межатомной связи $\left.\mathrm{C}-\mathrm{O} l=1.16 \cdot 10^{-10} \mathrm{~m}\right)$ имеет вид

$$
\bar{H}=\bar{T}+\bar{\Pi},
$$

где кинетическая энергия

$$
\bar{T}=\frac{1}{2}\left\{2 \dot{\bar{\vartheta}}_{1}^{2}+2 M_{2} \dot{\bar{\vartheta}}_{2}^{2}+2 M_{2} \dot{\bar{\vartheta}}_{3}^{2}\right\}
$$

потенциальная энергия

$$
\begin{aligned}
& \bar{\Pi}=\frac{1}{2}\left\{\sigma^{2}\left[K_{\mathrm{CO}}\left(\Delta \bar{r}_{12}^{2}+\Delta \bar{r}_{23}^{2}\right)+2 K_{\mathrm{OO}} \Delta \bar{r}_{12} \Delta \bar{r}_{23}\right]\right. \\
& \left.+\frac{1}{2 M_{2}} K_{\varphi \varphi} \Delta \varphi^{2}\right\}+\frac{1}{6 m_{E}}\left\{l ^ { 3 } \left[k_{r r r}\left(\Delta \bar{r}_{12}^{3}+\Delta \bar{r}_{23}^{3}\right)\right.\right. \\
& \left.+3 k_{r r r^{\prime}} \Delta \bar{r}_{12} \Delta \bar{r}_{23}\left(\Delta \bar{r}_{12}+\Delta \bar{r}_{23}\right)\right]+3 l k_{r \varphi \varphi} \\
& \left.\times\left(\Delta \bar{r}_{23}-\Delta \bar{r}_{12}\right) \Delta \varphi^{2}\right\}+\frac{1}{24 m_{E}}\left\{l ^ { 4 } \left[k_{r r r r}\left(\Delta \bar{r}_{12}^{4}+\Delta \bar{r}_{23}^{4}\right)\right.\right. \\
& \left.+4 k_{r r r r^{\prime}} \Delta \bar{r}_{12} \Delta \bar{r}_{23}\left(\Delta \bar{r}_{12}^{2}+\Delta \bar{r}_{23}^{2}\right)+6 k_{r r r^{\prime} r^{\prime}} \Delta \bar{r}_{12}^{2} \Delta \bar{r}_{23}^{2}\right] \\
& +6 l^{2}\left[k_{r r \varphi \varphi}\left(\Delta \bar{r}_{12}^{2}+\Delta \bar{r}_{23}^{2}\right)+2 k_{r r^{\prime} \varphi \varphi} \Delta \bar{r}_{12} \Delta \bar{r}_{23}\right] \Delta \varphi^{2} \\
& \left.+k_{\varphi \varphi \varphi \varphi} \Delta \varphi^{4}\right\} .
\end{aligned}
$$


Здесь $\Delta \bar{r}_{12}=\left(\bar{r}_{12}-1\right), \Delta \bar{r}_{23}=\left(\bar{r}_{23}-1\right)-$ удлинения межатомных связей; $\bar{r}_{i j}=\sqrt{\bar{X}_{i j}^{2}+\bar{Y}^{2}}-$ расстояние между $i$-м и $j$-м атомами $\left(\bar{X}_{12}=-\bar{\vartheta}_{1}+M_{2} \bar{\vartheta}_{3}-1\right.$, $\left.\bar{X}_{23}=\bar{\vartheta}_{1}+M_{2} \bar{\vartheta}_{3}+1, \bar{Y}=M_{2} \bar{\vartheta}_{2}\right) ; \Delta \varphi=\arctan \left(\bar{Y} / \bar{X}_{12}\right)-$ $-\arctan \left(\bar{Y} / \bar{X}_{23}\right)-$ изменение валентного угла при деформационных колебаниях. В дополнение к [3] введены обозначения: $k_{\mathrm{CO}}, k_{\mathrm{OO}}-$ не зависящие от удлинений слагаемые жесткостей пружин, моделирующих межатомные взаимодействия $\mathrm{C}-\mathrm{O}$ и $\mathrm{O}-\mathrm{O}$ соответственно; $k_{\varphi \varphi}-$ постоянная часть изгибной жесткости молекулы; $K_{\mathrm{CO}}=k_{\mathrm{CO}} / k_{q}, \quad K_{\mathrm{OO}}=k_{\mathrm{OO}} / k_{q}, \quad K_{\varphi \varphi}=k_{\varphi \varphi} / k_{\varphi} \quad-$ отношения перечисленных величин к коэффициентам модели [3], определенным через частоты [2] согласно [4]; $k_{r r r}, k_{r r r^{\prime}}, k_{r \varphi \varphi}, k_{r r r r}, k_{r r r r^{\prime}}, k_{r r r^{\prime} r^{\prime}}, k_{r r \varphi \varphi}, k_{r r^{\prime} \varphi \varphi}, k_{\varphi \varphi \varphi \varphi}-$ прочие коэффициенты разложений, обозначенные (за исключением , $k^{\text {“ }}$ и угла $\varphi$ ), согласно ссылающейся на [5] обзорной работе [6], и принятые, как и $k_{\mathrm{CO}}$, $k_{\text {Oо, }} k_{\varphi \varphi}$, по данным [5]; $m_{E}=k_{q} l^{2} / \sigma^{2}-$ масштаб энергии. Дополнительные по сравнению с [3] слагаемые в разложении (3) потенциала, характеризующие физический ангармонизм колебаний, подчеркнуты.

Анализ характерной зависимости „сила-удлинение“(рис. 2), показывает, что ангармонические эффекты начинают проявляться при деформациях межатомных связей более 0.2 .

Так как разброс экспериментальных данных в [6] по коэффициентам слагаемых четвертой степени в (3) достаточно велик, в дальнейшем анализе (кроме хаотических колебаний) учтены только кубичные слагаемые потенци ала. Заметим также, что взаимодействие „дальних соседей“ $\mathrm{O}-\mathrm{O}$, основная часть которого учитывается коэффициентом $K_{\mathrm{OO}}=k_{\mathrm{OO}} / k_{q}=7.3 \cdot 10^{-2}$, достаточно слабое.

Анализ численного решения уравнений движения в форме уравнений Лагранжа второго рода (уравнения с точностью до квадратов деформаций имеют вид

$$
\begin{gathered}
\ddot{\bar{\vartheta}}_{1}+\frac{\sigma^{2}}{2}\left[-K_{\mathrm{CO}} \bar{\xi}_{123,1}^{-}+K_{\mathrm{OO}} \bar{\xi}_{231.1}^{-}\right]+\frac{\bar{Y} \bar{r}_{D 123.2}^{+} \Delta \varphi}{4 M_{2}} \\
+\frac{\sigma^{2}}{4}\left\{-K_{r r r} \bar{\xi}_{123.2}^{-}+K_{r r r} \bar{R}^{-}+K_{r \varphi \varphi} \bar{\Phi}^{+}\right\}=0, \\
\ddot{\bar{\vartheta}}_{2}+\frac{\sigma^{2}}{2}\left(K_{\mathrm{CO}} \bar{D}_{r 123.1}^{+}+K_{\mathrm{OO}} \bar{D}_{r 231.1}^{+}\right) \bar{Y}+\frac{\bar{X}_{r 123.2}^{-} \Delta \varphi}{4 M_{2}} \\
+\frac{\sigma^{2}}{4}\left\{K_{r r r} \bar{D}_{r 123.2}^{+} \bar{Y}+K_{r r r} \bar{R}_{\bar{Y}}+K_{r \varphi \varphi} \bar{\Phi}_{\bar{Y}}\right\}=0, \\
\ddot{\bar{\vartheta}}_{3}+\frac{\sigma^{2}}{2}\left[K_{\mathrm{CO}} \bar{\xi}_{123.1}^{+}+K_{\mathrm{OO}} \bar{\xi}_{231.1}^{+}\right]-\frac{\bar{Y}-r_{D 123.2}^{-} \Delta \varphi}{4 M_{2}} \\
+\frac{\sigma^{2}}{4}\left\{K_{r r r} \bar{\xi}_{123.2}^{+}+K_{r r r} \bar{R}^{+}-K_{r \varphi \varphi} \bar{\Phi}^{-}\right\}=0,
\end{gathered}
$$

где

$$
\begin{gathered}
\bar{\xi}_{123, p}^{ \pm}=\bar{X}_{r, 12} \Delta \bar{r}_{12}^{p} \pm \bar{X}_{r, 23} \Delta \bar{r}_{23}^{p}, \\
\bar{R}^{ \pm}=\bar{X}_{r, 23} \Delta \bar{r}_{12}^{2} \pm 2 \bar{X}_{r 123.1}^{ \pm} \Delta \bar{r}_{12} \Delta \bar{r}_{23} \pm \bar{X}_{r, 12} \Delta \bar{r}_{23}^{2},
\end{gathered}
$$

$$
\begin{gathered}
\bar{R}_{\bar{Y}}=\Delta \bar{r}_{12}^{2} / \bar{r}_{23}+2 \bar{r}_{D 123.1}^{+} \Delta \bar{r}_{12} \Delta \bar{r}_{23}+\Delta \bar{r}_{23}^{2} / \bar{r}_{12}, \\
\bar{\Phi}^{ \pm}=\bar{X}_{r 123.1}^{ \pm} \Delta \varphi^{2}+2 \bar{Y}_{D 123.2}^{ \pm}\left(\Delta \bar{r}_{23}-\Delta \bar{r}_{12}\right) \Delta \varphi, \\
\bar{\Phi}_{\bar{Y}}=2 \bar{X}_{r 123.2}^{-}\left(\Delta \bar{r}_{23}-\Delta \bar{r}_{12}\right) \Delta \varphi-\bar{Y}_{\bar{r}_{123.1} \Delta \varphi^{2},} \\
\bar{\xi}_{231, p}^{ \pm}=\bar{X}_{r, 23} \Delta \bar{r}_{12}^{p} \pm \bar{X}_{r, 12} \Delta \bar{r}_{23}^{p}, \\
\bar{X}_{r, i j}=\bar{X}_{i j} / \bar{r}_{i j}, \\
\bar{r}_{D 123, p}^{+}=1 / \bar{r}_{12}^{p} \pm 1 / \bar{r}_{23}^{p}, \\
\bar{X}_{r 123.1}^{ \pm}=\bar{X}_{12} / \bar{r}_{12}^{p} \pm \bar{X}_{23} / \bar{r}_{23}^{p}, \\
\bar{D}_{r 123, p}^{ \pm}=\Delta \bar{r}_{12}^{p} / \Delta \bar{r}_{12} \pm \Delta \bar{r}_{23}^{p} / \bar{r}_{23}, \\
\bar{D}_{r 231, p}^{ \pm}=\Delta \bar{r}_{12}^{p} / \bar{r}_{23} \pm \Delta \bar{r}_{23}^{p} / \bar{r}_{12}, \\
K_{r r r}=k_{r r r} /\left(k_{q} / l\right), \\
K_{r r r^{\prime}}=k_{r r r^{\prime}} /\left(k_{q} / l\right), \\
\left.K_{r \varphi \varphi}=k_{r \varphi \varphi} /\left(k_{q} l\right), \quad p \in Z\right)(\text { puc. 3), }
\end{gathered}
$$

позволяет сделать следующие выводы:

1) физическая нелинейность практически ничего не привносит не только в качественную, но и в количественную составляющую картины явления, так что ее учет может быть целесообразным только при уточнении интенсивностей слабых линий как гармоник решения (что будет показано ниже в разд. 3);

2) при малых колебаниях и начальных условиях, соответствующих наивысшей интенсивности обмена энергией между степенями свободы, антисимметричные $\vartheta_{3}(t)$ колебания не возбуждаются симметричными $\vartheta_{1}(t)$ и деформационными $\vartheta_{2}(t)$. Следовательно, приняв в нашем случае $\left\{\bar{\vartheta}_{20} \ll \bar{\vartheta}_{10}, \bar{\vartheta}_{10} \ll 1\right\} ; \bar{\vartheta}_{30}=0, \dot{\bar{\vartheta}}_{30}=0$, мы при поиске приближенного аналитического решения исключаем из рассмотрения антисимметричное колебание.

Для более детального анализа рассмотрим зависимость максимального абсолютного значения отклонения
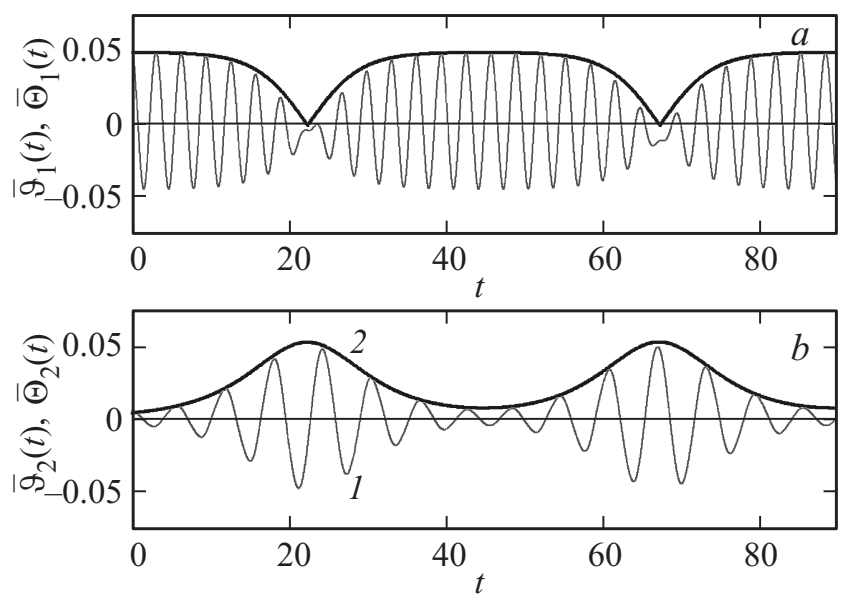

Рис. 3. Сопоставаление численного (кривые 1) и аналитического в первом приближении (кривые 2 - огибающие (7)) решения уравнений (4). 
по антисимметричной координате $\max \left(\left|\bar{\vartheta}_{3}\right|\right)$ от $\bar{\vartheta}_{10}$. Расщепление линий спектра наиболее интенсивно проявляется в асимптотическом случае, поэтому решение проведем при начальных условиях $\bar{\vartheta}_{20} \ll \bar{\vartheta}_{10}, \bar{\vartheta}_{30}=0$, варьируя величину $\bar{\vartheta}_{10}$ и оставляя неизменным ее отношение к $\bar{\vartheta}_{20}$, равное 10 . Результаты анализа, представленные на рис. 4 , показывают наличие критической величины $\left(\bar{\vartheta}_{10}\right)_{\text {сr }} \approx 0.25$, после которой влияние $\bar{\vartheta}_{3}$ на резонанс Ферми становится существенным.

Можно показать, что антисимметричное возмущение воздействует на симметричное и изгибное (деформационное) движения молекулы аналогичным образом. На pис. 5, $a$ представлены кривые, позволяющие проанализировать устойчивость симметричных и деформационных колебаний молекулы к антисимметричному начальному отклонению $\bar{\vartheta}_{30}$; все начальные импульсы приняты нулевыми. Критическая величина $\bar{\vartheta}_{30}$ явно коррелирует с $\left(\bar{\vartheta}_{10}\right)_{\text {cr }}$ на рис. 4: их отношение приблизительно соответствует отношению $\bar{\vartheta}_{20} / \bar{\vartheta}_{10}$, принятому на предыдущем этапе моделирования, результаты которого представлены на рис. 4. Когда амплитуды колебаний по координате $\bar{\vartheta}_{30}$ малы, обмен энергией между симметричной и антисимметричной формами проявляется слабо (рис. $5, b$, для сравнения на рис. $4, b$ приводится огибающая решения в первом приближении $\bar{\Theta}_{3}(t)=\bar{\vartheta}_{30}$, которая будет получена в следующем разделе методом нормальной формы с применением алгоритма инвариантной
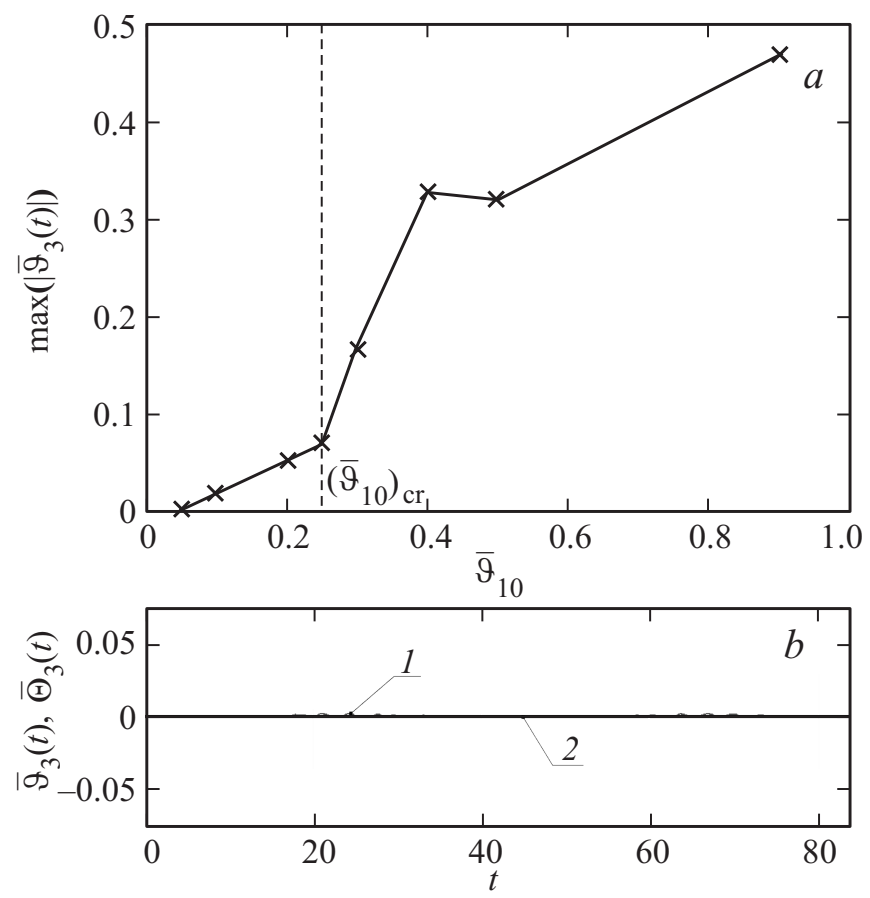

Рис. 4. Характеристики возбуждения антисимметричного колебания совокупным действием симметричного и деформационного начальных отклонений: $a-$ зависимость $\max \left(\left|\bar{\vartheta}_{3}(t)\right|\right)$ от $\bar{\vartheta}_{10}, b-$ реализация колебательного процесса (численное моделирование) в условиях рис. 3: 1 - расчетная кривая, 2 - огибающая (7).

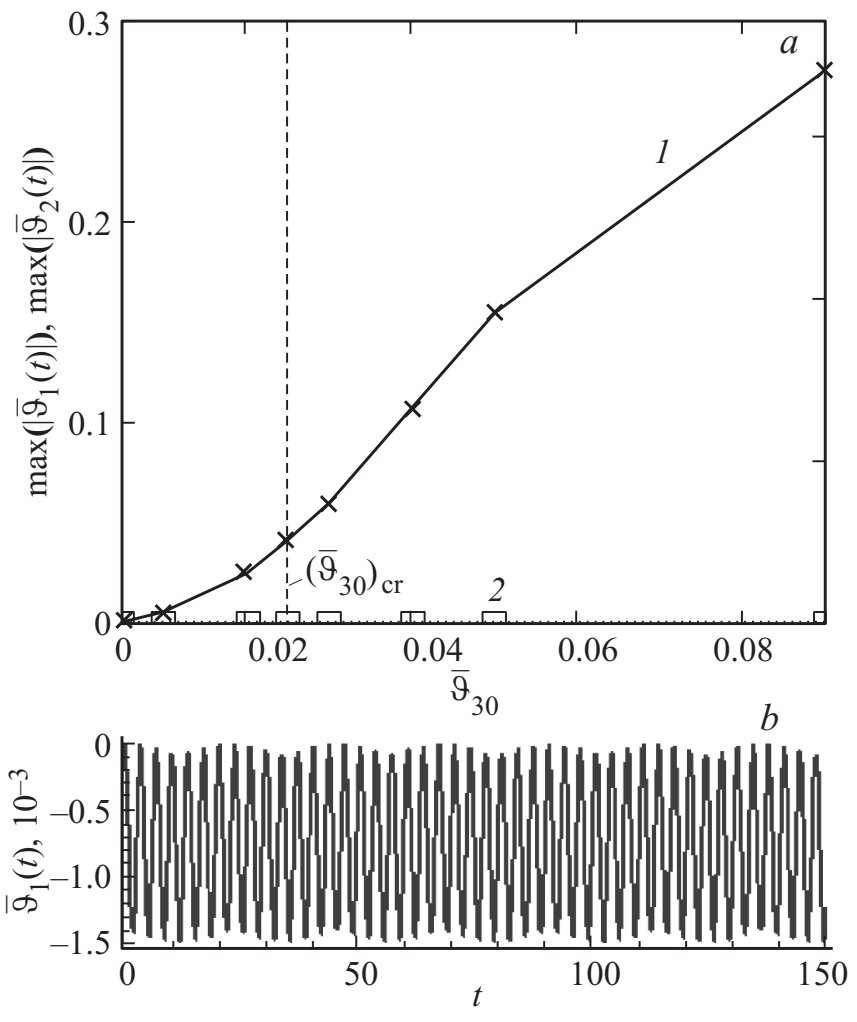

Рис. 5. Характеристики возбуждения симметричного и деформационного колебаний антисимметричным начальным отклонением: $a$ - зависимости $\max \left(\left|\bar{\vartheta}_{1}(t)\right|\right)$ (кривая 1), $\max \left(\left|\bar{\vartheta}_{2}(t)\right|\right)$ (кривая 2) от $\bar{\vartheta}_{30}\left(\bar{\vartheta}_{10}=0, \bar{\vartheta}_{20}=0\right), b$ - реализация колебательного процесса (численное моделирование) при начальных условиях $\bar{\vartheta}_{30}=5 \cdot 10^{-3}, \bar{\vartheta}_{j 0}=0, \dot{\bar{\vartheta}}_{n 0}=0(j=1,2$, $n=1, \ldots, 3)$.

нормализации [7]). При указанных начальных условиях колебания происходят вдоль оси недеформированной молекулы, так что изгибное колебание с частотой $v_{2}$ не возбуждается. Однако в силу возможной неустойчивости деформационного колебания, даже малое начальное отклонение $\bar{\vartheta}_{20}$ может привести к его раскачке.

Э. Ферми [4] (а вслед за ним и авторы настоящей работы $[3,8])$ полагал, что антисимметричное колебание не влияет на взаимодействие симметричного с деформационным. Полученные результаты позволяют установить границы применимости - малые амплитуды колебаний, при которых движение носит регулярный характер.

\section{2. Аналитическое исследование процесса обмена энергией между степенями свободы}

Применим к анализу метод приведения системы уравнений к рекуррентной нормальной форме ПуанкареБиркгофа по алгоритму инвариантной нормализации в редакции В.Ф. Журавлева-А.Г. Петрова [7]. Нормировкой приведем гамильтониан (1) к интегрируемому 
виду [3]

$$
\begin{aligned}
\frac{m_{t}^{2}}{m_{H}}= & \bar{H}=\bar{H}_{2}+\bar{H}_{3} \\
= & \frac{1}{2}\left\{\left[\bar{\psi}_{1}^{2}+4 \bar{q}_{1}^{2}\right]+\left[\bar{\psi}_{2}^{2}+\tilde{\bar{\Omega}}_{2}^{2} \bar{q}_{2}^{2}\right]+\left[\bar{\psi}_{3}^{2}+\tilde{\bar{\Omega}}_{3}^{2} \bar{q}_{3}^{2}\right]\right\} \\
& +\tilde{k}_{111} q_{1}^{3}+\frac{3}{2} \bar{q}_{1} \bar{q}_{2}^{2}+\tilde{k}_{133} \bar{q}_{1} \bar{q}_{3}^{2}+\tilde{k}_{322} \bar{q}_{3} \bar{q}_{2}^{2}+\tilde{\mu} \bar{q}_{1}^{2},
\end{aligned}
$$

где $\tilde{t}=t / m_{\tilde{t}}-$ нормированное время, $\bar{q}_{j}=\bar{\vartheta}_{j} / m_{j}, \bar{\psi}_{j}=$ $=\operatorname{dot} \bar{q}_{j}=d \bar{q}_{j} / d \tilde{t}(j=1, \ldots, 3)-$ нормированные координаты и скорости, $m_{\tilde{t}}=1 / \sqrt{K_{\mathrm{CO}}+K_{\mathrm{OO}}}=0.990, m_{1}=S, m_{2}=$ $=m_{3}=S / \sqrt{M_{2}}, m_{H}=2 S^{2}-$ нормировочные множители времени, координат и гамильтониана соответственно (здесь введено обозначение $\left.S=3 M_{2}\left(K_{\mathrm{CO}}+K_{\mathrm{OO}}\right) / k_{122}\right)$, $\tilde{k}_{111}=\left(m_{t}^{2} m_{1}^{3} / m_{H}\right) k_{111}, \tilde{k}_{113}=\left(m_{\tilde{t}}^{2} m_{1} m_{3}^{2} / m_{H}\right) k_{133}, \quad \tilde{k}_{322}=$ $=\left(m_{\tilde{t}}^{2} m_{3} m_{2}^{2} / m_{H}\right) / k_{322}, \quad \tilde{\mu}=4\left(K_{\mathrm{CO}}+K_{\mathrm{OO}}\right)\left(m_{\tilde{t}} m_{1}^{2} / m_{H}\right) \mu$, $k_{111}=\sigma^{2} / 3\left(k_{r r r}+3 k_{r r r^{\prime}}\right) l / k_{q}<0, \quad k_{122}=\sigma^{2} M_{2}^{2}\left[\left(K_{\mathrm{CO}}+\right.\right.$ $\left.\left.+K_{\mathrm{OO}}\right)-2 / M_{2} K_{\varphi \varphi}+2 / 300\left(l k_{r r r} / k_{q}\right)\right]>0$ (т. е. коэффициент $k_{r \varphi \varphi}$ разложения по параметрам физической нелинейности, несмотря на аналогию с множителем перед $\bar{q}_{1} \bar{q}_{2}^{2}$ в разложении (5) по параметрам геометрической не линейности в кубичной части потенциала не влияет на характеристики процесса обмена энергией между модами),

$$
\begin{aligned}
k_{133} & =\sigma^{2} M_{2}^{2} l\left(k_{r r r}-k_{r r r^{\prime}}\right) / k_{q}<0 . \\
k_{322} & =4 \sigma^{2} M_{2}^{3}\left(k_{r \varphi \varphi} / k_{q l}\right) \\
& =4 \sigma^{2} M_{2}^{3}\left\{k_{r \varphi \varphi} /\left[\left(2 M_{2} \sigma^{2} / t^{2}\right) k_{\varphi} l\right]\right\} \\
& =2 M_{2}^{2}\left(l k_{r \varphi \varphi} / k_{\varphi}\right)<0 .
\end{aligned}
$$

Действительно, с точностью до кубов деформаций в (3) пружины, моделирующие межатомные взаимодействия, „мягкие“, а физический ангармонизм мало влияет на коэффициент в резонансном слагаемом $k_{122}$, ответственный за период обмена энергией между модами. Нормировка времени введена для учета отклонений суммарной относительной постоянной части коэффициента жесткости связей $\mathrm{C}-\mathrm{O}$ и $\mathrm{O}-\mathrm{O}$ на растяжениесжатие $\left(K_{\mathrm{CO}}+K_{\mathrm{OO}}\right)=1.020$ от единицы. В формуле (1) она не вводится, чтобы показать влияние различных составляющих жесткости $\left(k_{\mathrm{CO}}, k_{\mathrm{OO}}, k_{\varphi \varphi}\right)$ на частоты колебаний линеаризованной системы. Главные нормированные частоты будут при этом соответственно равны $\tilde{\bar{\Omega}}_{1}=\bar{\Omega}_{1} / m_{\tilde{\omega}}=\bar{\Omega}_{1} m_{\tilde{t}}=\sigma=2+\mu, \tilde{\bar{\Omega}}_{1}=$ $=\bar{\Omega}_{2} m_{\tilde{t}}=\sqrt{K_{\varphi \varphi}} m_{\tilde{t}}=1.005 \cdot 990=0.99 \approx 1, \tilde{\bar{\Omega}}_{3}=\bar{\Omega}_{3} m_{\tilde{t}}=$ $=\left(K_{\mathrm{CO}}-K_{\mathrm{OO}}\right) \sqrt{11 / 3}(2+\mu) m_{\hat{t}} \approx 2 \sqrt{11 / 3} \approx 4$. Расстройка частот как $\left(\tilde{\bar{\Omega}}_{1} / \tilde{\bar{\Omega}}_{2}-2\right)$ не вводится, чтобы не внести погрешности эксперимента [5] в значения частот $v_{1}=1338 \mathrm{sm}^{-1}$ и $v_{2}=667 \mathrm{sm}^{-1}[2]$.

Для учета расстройки частот $v_{1}$ и $v_{2}$ от целочисленного соотношения $2: 1 \mu=\sigma-2=v_{1} / v_{2}-2$ можно, как и в $[8]$ (кубичная часть гамильтониана $\bar{H}_{3}$ аналогична [8]; слагаемые, содержащие $\bar{q}_{1}^{3}$ и $\bar{q}_{1} \bar{q}_{3}^{2}$, а также $\bar{q}_{3} \bar{q}_{2}^{2}$ (что является новым по сравнению с [3] и $[8])$, не влияют на нормальную форму первого приближения), проследить аналогию с [9], что приводит к асимптотическому решению на интервале $t \in[0 ; n T]$ (при начальных отклонениях $\bar{\vartheta}_{10}=0.05, \bar{\vartheta}_{20}=0.1 \bar{\vartheta}_{10}$ и нулевых начальных импульсах $T=44.612-$ период обмена („перекачки“ $[7,9]$ ) энергией между симметричной и деформационной степенями свободы) в виде суммы $n$-солитонов

$$
\begin{aligned}
& \bar{\vartheta}_{1 a}(t)=\bar{\Theta}_{1}(t) \cos 2 t, \bar{\vartheta}_{2 a}(t)=\bar{\Theta}_{2}(t) \cos t, \\
& \bar{\vartheta}_{3 a}(t)=\bar{\Theta}_{3}(t) \cos \bar{\Omega}_{3} t,
\end{aligned}
$$

где огибающие

$$
\begin{gathered}
\bar{\Theta}_{1}(t)=\bar{\vartheta}_{10}\left\{\sum_{i=1}^{n}\left|\tanh \left[\tau(t)-(1+2(i-1)) \frac{\tau_{0}}{2}\right]\right|-(n-1)\right\}, \\
\bar{\Theta}_{2}(t)=\frac{2}{\sqrt{M_{2}}} \bar{\vartheta}_{10} \sum_{i=1}^{n} \operatorname{sech}\left[\tau(t)-(1+2(i-1)) \frac{\tau_{0}}{2}\right], \\
\bar{\Theta}_{3}(t)=\bar{\vartheta}_{30} .
\end{gathered}
$$

Как следует из анализа основных соотношений алгоритма нормальной формы [7], первое приближение не содержит антисимметричного колебания именно из-за значительного отличия его частоты от двух других частот, между которыми возникает внутренний резонанс третьего порядка, что отмечал еще Э. Ферми [4]. Сопоставление аналитического решения (6) с численным (рис. 3) показывает хорошее их согласие (так, период биений численного решения для координаты $\bar{\vartheta}_{1}$ составляет $T_{1}=44.903$, а для $\bar{\vartheta}_{2}-T_{2}=44.573$; среднеарифметическое значение $T_{12 m}=\left(T_{1}+T_{2}\right) / 2=44.738$. Физически в силу закона сохранения энергии должно выполняться равенство $T_{1}=T_{2}=T$. Разность $\left(T_{1}-T_{2}\right) \ll T_{1}, T_{2}$, что косвенно указывает на высокую точность расчетов). Некоторые отличия, по всей видимости, вызваны вводом расстройки по спектроскопическим данным [2] (наличие антисимметриной координаты, взаимодействующей, согласно рис. $4, b$, с симметричной и деформационной, в слагаемом $\left[l^{3} /\left(6 m_{E}\right)\right] k_{r r r}\left(\Delta \bar{r}_{12}^{3}+\Delta \bar{r}_{23}^{3}\right)$ потенциала (3), учтено (в отличие от [8] кубичная нелинейность деформирования пружин влияет на период перекачки; случай отсутствия бокового отклонения (соответствующих начальных условий) не рассматривается)). Данный вопрос требует дополнительного исследования.

\section{3. Спектральный анализ}

В условиях наиболее существенного проявления „перекачки“ энергии между степенями свободы решение определяющих уравнений, соответствующих гамильтониану (1), как численное, так и асимптотическое (6) обладает свойством периодичности, что позволяет разложить его в ряд Фурье. Наибольший вклад в эту сумму 


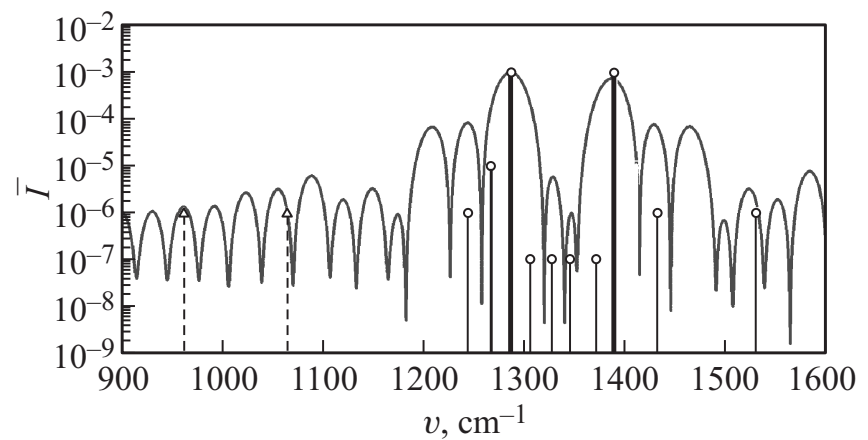

Рис. 6. Область спектра численного решения полных уравнений в окрестности частоты симметричного колебания $v_{1}$ при начальных условиях, соответствующих рис. 3 .

будут вносить гармоники с частотами, не слишком сильно отличающимися от основных.

Полученный таким образом спектр интенсивностей гармоник симметричного колебания $\bar{\vartheta}_{1}(t)$ показан на рис. 6. Будучи совмещенным с экспериментальными линиями [2] (безразмерные интенсивности последних принимались из качественных соображений: очень сильная, сильная, средняя, слабая и очень слабая линии, и показаны на рис. 6 вертикальными линиями: сплошными линиями - комбинационные, штриховые - инфракрасные. Толщина и высота линии качественно соответствуют ее безразмерной интенсивности) он позволяет идентифицировать большее число частот по сравнению с линейным [3] и квадратичным [8] приближениями для сил упругости.

\section{4. К вопросу о нерегулярных режимах движения}

Известно, что в динамических системах при наличии двух и более положений устойчивого равновесия, разделенных неустойчивыми положениями, или потенциальными барьерами, возможны хаотические режимы вынужденных колебаний, сопровождающиеся „перескоками“ между соседними положениями устойчивого равновесия [10]. Для систем со многими степенями свободы роль внешних нагрузок для выделенной нормальной координаты играют остальные, поэтому такое возможно в том числе и при условии автономности.

В соответствии с ранее высказанными замечаниями антисимметричное колебание $\bar{\vartheta}_{3}$ учитывать не будем. Оно не влияет $[3,8]$ на параметры регулярного режима периодического обмена энергией между степенями свободы молекулы; вопрос же его воздействия на режимы хаотические требует дополнительного исследования. Тогда становится возможным построить график потенциала (3) в пространстве двух аргументов $\bar{\vartheta}_{1}$ и $\bar{\vartheta}_{2}$, рис. 7.

Из анализа графика следует, что учет добавочного (в сравнении с физически линейной моделью $[3,11]$ ) ангармонизма мало сказывается на положении седловой точки, но влияет на величину максимума потенциала. Следовательно, воздействие новых ангармоничных членов должно проявляться в окрестности седловой точки. В частности, по этой причине при малых амплитудах колебаний (рис. 3,4 при $\bar{\vartheta}_{10} \leq 0.2$, рис. 5 при $\bar{\vartheta}_{30} \leq 0.02$, рис. 6) не учитывались слагаемые четвертого порядка в разложении (3), точность экспериментального определения (данных [6]) которых, как уже упоминалось в разд. 1, сравнительно невысока.

Раскладывая в ряд потенциал $\bar{\Pi}_{12}=\bar{\Pi}_{12}\left(\bar{\vartheta}_{1}, \bar{\vartheta}_{2}\right)$ с удержанием членов 4-го порядка, получаем

$$
\begin{aligned}
\bar{\Pi}_{12}\left(\bar{\vartheta}_{1}, \bar{\vartheta}_{2}\right)= & \bar{\Omega}_{1}^{2} \bar{\vartheta}_{1}^{2}+M_{2} \bar{\Omega}_{2}^{2} \bar{\vartheta}_{2}^{2}+k_{111} \bar{\vartheta}_{1}^{3}+k_{122} \bar{\vartheta}_{1} \bar{\vartheta}_{2}^{2} \\
& +k_{1111} \bar{\vartheta}_{1}^{4}+k_{1122} \bar{\vartheta}_{1}^{2} \bar{\vartheta}_{2}^{2}+k_{2222} \bar{\vartheta}_{2}^{4}+o\left(\varepsilon^{5}\right),
\end{aligned}
$$

где $k_{1111}=\left(\sigma^{2} / 12\right)\left[l^{2}\left(k_{r r r r}+4 k_{r r r r^{\prime}}+3 k_{r r r^{\prime} r^{\prime}}\right) / k_{q}\right]>0$,

$$
\begin{gathered}
k_{1122}=2 \sigma^{2} M_{2}^{2}\left[\left(k_{r r \varphi \varphi}+k_{r r^{\prime} \varphi \varphi}\right) / k_{q}\right]-\sigma^{2} M_{2}^{2}\left(K_{\mathrm{CO}}+K_{\mathrm{OO}}\right) \\
+3 M_{2} K_{\varphi \varphi}+\sigma^{2} M_{2}^{2} / 2\left[l\left(k_{r r r}+3 k_{r r r^{\prime}}\right) / k_{q}\right]<0 \\
k_{2222}=\left(\sigma^{2} / 4\right) M_{2}^{4}\left(\left(K_{\mathrm{CO}}+K_{\mathrm{OO}}\right)-2 / 3 M_{2}^{3} K_{\varphi \varphi}\right. \\
+(2 / 3) \sigma^{2} M_{2}^{4}\left(k_{\varphi \varphi \varphi \varphi} / k_{q} l^{2}\right)>0
\end{gathered}
$$

за малый параметр $\varepsilon$ принята единица.

Таким образом, двухьямочный характер $\bar{\Pi}_{12}\left(\bar{\vartheta}_{1}, \bar{\vartheta}_{2}\right)$ в аппроксимации (8) обусловлен только слагаемым, содержащим $\bar{\vartheta}_{1}^{2} \bar{\vartheta}_{2}^{2}$, что при использовании необходимого условия Мельникова возникновения хаоса $[12,13]$, требующегося в том числе для определения размеров хаотической области в фазовом пространстве, не позволяет выделить в качестве невозмущенных одностепенные парциальные

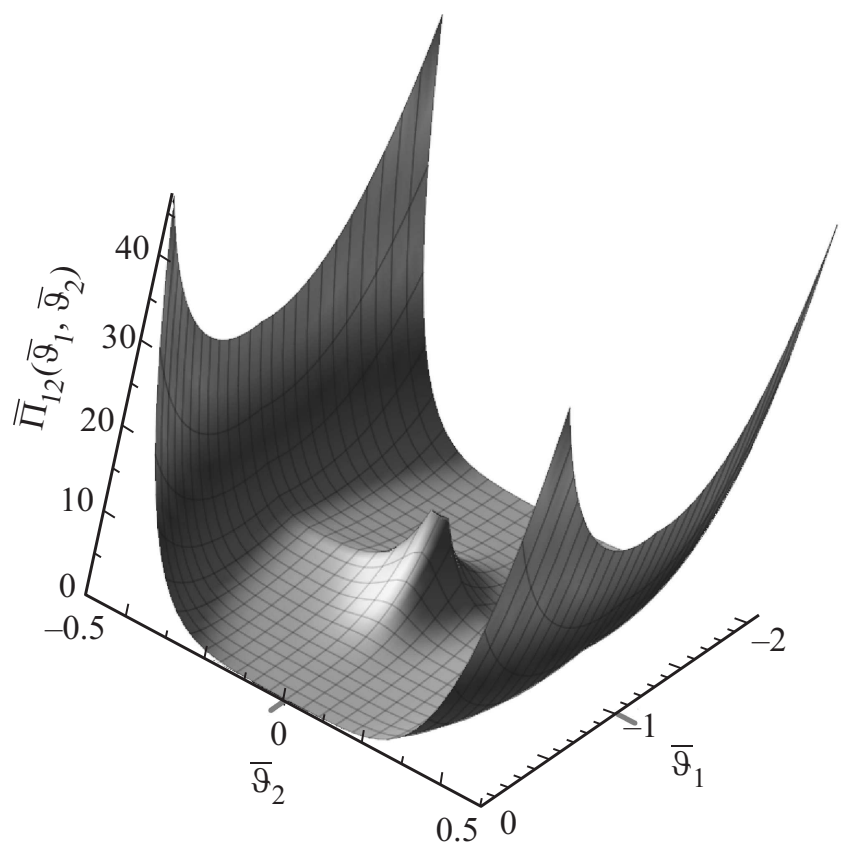

Рис. 7. Поверхность потенциала механической модели молекулы $\bar{\Pi}_{12}\left(\bar{\vartheta}_{1}, \bar{\vartheta}_{2}\right)=\bar{\Pi}\left(\bar{\vartheta}_{1}, \bar{\vartheta}_{2}, \bar{\vartheta}_{3}=0\right)$. 

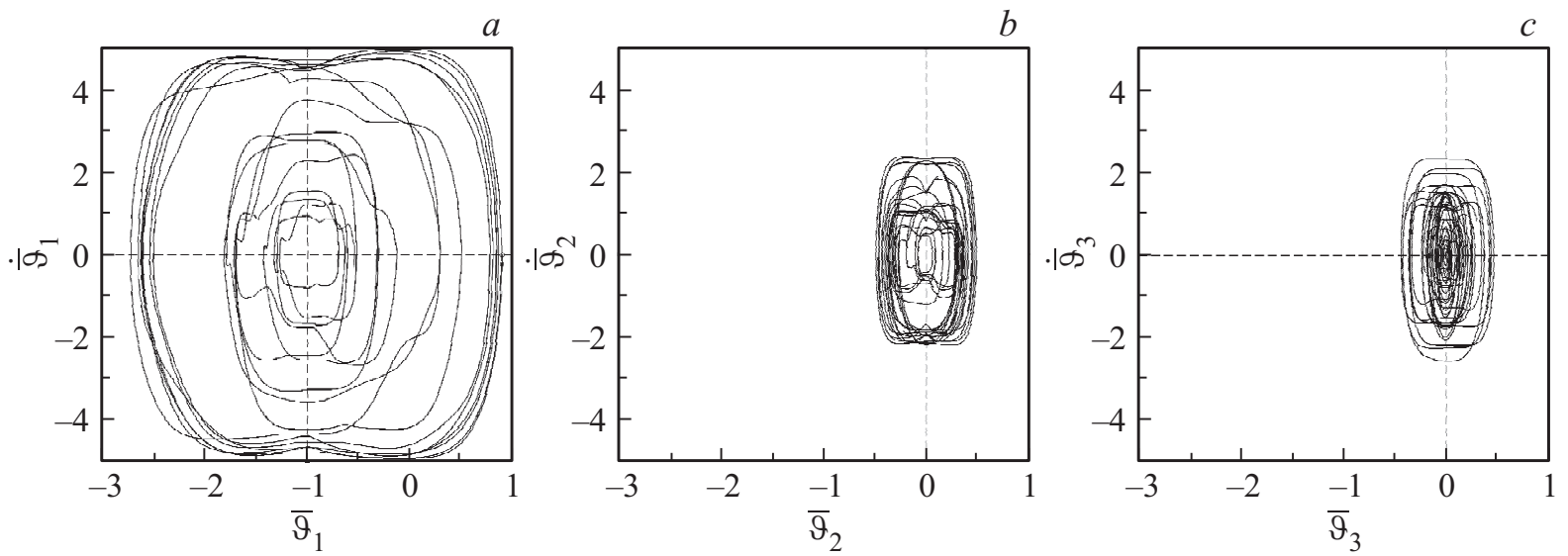

Рис. 8. Фазовые портреты $\left(a-\right.$ симметричного колебания $\bar{\vartheta}_{1}, b-$ деформационного $\bar{\vartheta}_{2}$ и $c-$ антисимметричного $\left.\bar{\vartheta}_{3}\right)$ при начальных условиях $\bar{\vartheta}_{10}=0.9, \bar{\vartheta}_{20}=0.09, \bar{\vartheta}_{30}=0, \dot{\bar{\vartheta}}_{10}=0, \dot{\bar{\vartheta}}_{20}=0, \dot{\bar{\vartheta}}_{30}=0$.
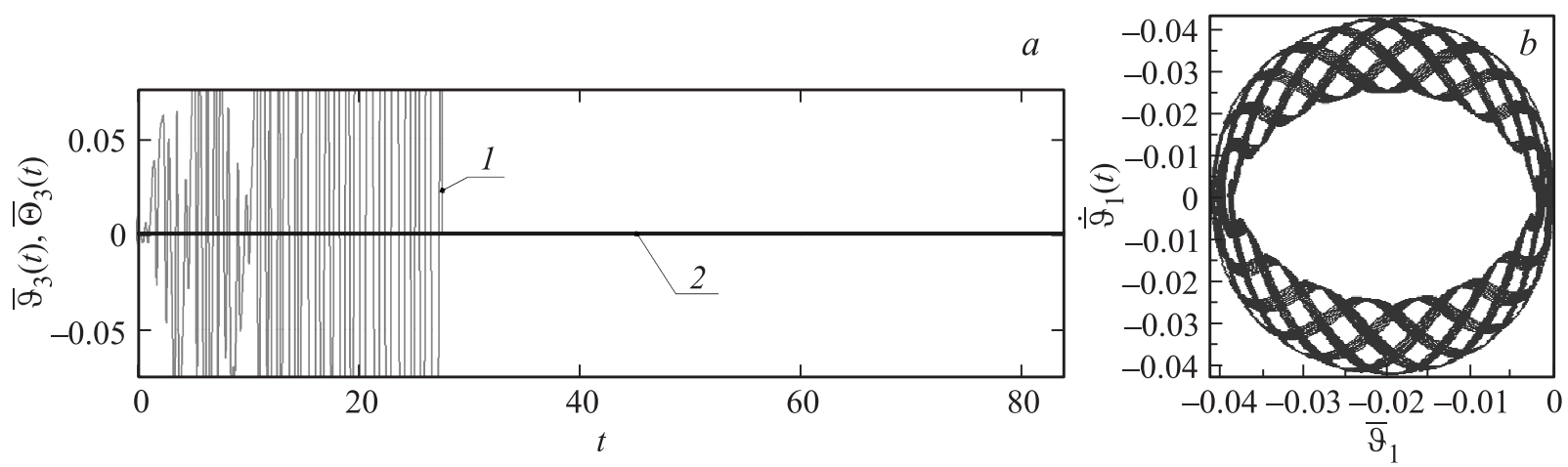

Рис. 9. Характерные реализации хаотического колебательного процесса: $a$ - график $\bar{\vartheta}_{3}(t)$ в условиях рис. 8 (время счета до 27.630 единиц безразмерного времени $), b-$ фазовый портрет $\left(\bar{\vartheta}_{30}=2.5 \cdot 10^{-2}, \bar{\vartheta}_{j 0}=0, \dot{\bar{\vartheta}}_{n 0}=0(j=1,2 ; n=1, \ldots, 3)\right)$.

системы. Последнего требует известное $[14,15]$ обобщение метода Мельникова на случай автономных систем со многими степенями свободы.

Численное же моделирование позволяет обнаружить в системе стохастический аттрактор (рис. 8).

В случае соответствия начального запаса энергии потенциальной энергии в седловой точке взаимодействие симметричной и деформационной форм колебаний с антисимметричной возможно при любом отношении $\bar{\vartheta}_{30}$ к $\bar{\vartheta}_{10 \text { и }} \bar{\vartheta}_{20}$.

Так, антисимметричные колебания могут возбуждаться симметричными и деформационными при $\bar{\vartheta}_{10}$, большем критической величины $\left(\bar{\vartheta}_{10}\right)_{\mathrm{cr}}$, установленной в разд. 1. В качестве примера на рис. 9, $a$ представлена характерная зависимость $\bar{\vartheta}_{3}(t)$ в условиях рис. 8 (для сопоставления с рис. 3 масштабы и диапазоны переменных оставлены прежними), когда в системе возникает странный аттрактор.

Аналогично возбуждение симметричных колебаний антисимметричными возможно при $\bar{\vartheta}_{30}>\left(\bar{\vartheta}_{30}\right)_{\text {cr }}$, достаточном для попадания в окрестность седловой точки (рис. 9, $b$ ).
Сценарий перехода от регулярных колебаний к стохастическим является темой отдельного рассмотрения.

\section{Заключение}

В работе предложен основанный на методах классической динамики подход к исследованию нелинейных колебаний молекулы $\mathrm{CO}_{2}$. В приближении „дальних соседей“ получены уравнения колебаний молекулы, учитывающие ангармонизм валентных колебаний и силового поля молекулы („жесткость“ и „мягкость“ пружин). Численным моделированием и аналитическим решением уравнений методом инвариантной нормализации установлено, что основной вклад в возникновение резонанса Ферми вносит ангармонизм деформационных колебаний, влияние ангармонизма силового поля и учет связи атомов О-О в молекуле позволяет идентифицировать дополнительное число частот в спектре молекулы. Численным моделированием обнаружено возникновение странного аттрактора. Даны рекомендации по выбору начальных условий возбуждения колебаний в молекуле, предотвращающих возможность перехода ее регулярных колебаний в хаотические. 


\section{Список литературы}

[1] Волькенштейн М.В., Ельшевич М.А., Степанов Б.И. Колебания молекул. М., Л.: Гостехиздат, 1949. Т. 1. 600 с.; T. 2. $440 \mathrm{c}$.

[2] Герцберг Г. Колебательные и вращательные спектры многоатомных молекул. М.: ИИЛ, 1949. 647 с.

[3] Алдошин Г.Т., Яковлев С.П. // Изв. РАН. МТТ. 2015. № 32. C. $42-53$.

[4] Fermi E. // Zs. für Physik. 1931. N 71. P. 250-259.

[5] Lacy M. // Molec. Phys. 1982. Vol. 45. N 2. P. 253-258.

[6] Attila G. Császàr // J. Phys. Chem. 1992. Vol. 96. P. 78987904.

[7] Петров А. Г. // Изв. РАН. МТТ. 2006. № 5. С. 18-28.

[8] Алдошин Г.Т., Яковлев С.П. // ХІ Всерос. съезд по фундаментальным проблемам теоретической и прикладной механики: труды съезда. Казань, 20-24 августа 2015 г. Казань: Изд-во Казанского (Приволжского) федерального ун-та, 2015. 4480 с. С. 109-111.

[9] Петров А. Г., Шундерюк А. М. // Изв. РАН. МТТ. 2010. № 2. C. $27-40$.

[10] Алдошин Г.Т. Теория линейных и нелинейных колебаний: учебное пособие. СПб.: Лань, 2013. 320 с.

[11] Алдошин Г.Т., Яковлев С.П. // International Conference on Mechanics - Seventh Polyakhov's Reading. 2015. P. 1-4.

[12] Симиу Э. Хаотические переходы в детерминированных и стохастических системах. Применение метода Мельникова в технике, физике и нейрофизиологии / Пер. с англ. под ред. А.С. Ковалевой. М.: Физматлит, 2007. 208 с.

[13] Автореф. канд. дис. Ледков А.С. Исследование резонансных движений сегментально-конических тел в атмосфере. Самара, 2009. 149 c.

[14] Perko L.M. // Rocky Mountain Journal of Mathematics. 1992. Vol. 22. N 3. P. $980-988$.

[15] Jerrold E. Marsden and Tudor S. Ratiu. Introduction to Mechanics and Symmetry: A Basic Exposition of Classical Mechanical Systems. Second Edition, 1998. 549 p. 